\title{
An Efficient Adaptive Channel Allocation Scheme For Cellular
} Networks

\author{
R R Kar ${ }^{1}$, S S Nayak ${ }^{2}$ \\ ${ }^{1}$ Dept. of Computer Science and Engineering/ Centurion University of Technology and Management, \\ Paralakhemundi, Odisha, India \\ ${ }^{2}$ Dept. of Physics/ Centurion University of Technology and Management, Paralakhemundi, Odisha, India
}

\begin{abstract}
Cellular networking is providing global information access to users on the move. Handoff algorithms allow the users to move among heterogeneous wireless networks. In this paper, the authors have proposed an efficient Adaptive Channel Allocation Scheme (ACAS) where the number of guard channels is adjusted automatically based on the average handoff blocking rate measured in the past certain period of time. The handoff blocking rate and the new call blocking rates are minimized. It is seen that the proposed scheme is far efficient than the Static Channel Allocation Scheme. The resource utilization of the proposed scheme is more.
\end{abstract}

Keywords: Handoff, Guard Channel, Adaptive Channel, Fixed Channel, ACAS, Spectrum

\section{INTRODUCTION}

There is worldwide growth of cellular telephony. Users are giving more importance to portability as a service feature. As users move from one location to another require the communication path to provide sufficient buffers that cater to the change of bandwidth. Cellular systems deploy smaller cells in order to achieve high system capacity due to the limited spectrum. The frequency band is divided into smaller bands and those bands are reused in non-interfering cells [2].

In a cellular network the subscribers can wander anywhere in the region and remain connected to the Public Switched Telephone Networks (PSTN) via their wireless mobile devices. Mobile Stations (MS), Base Station (BS) and Mobile Switching Centre (MSC) constitute a cellular network. The base station covers each cell. It covers area of few kilometers in diameter. A base station serves each cell. A group of BSs is connected to MSC. A group of MSC is then connected to the MSC of other groups and then to PSTN. The MSC stores information about the subscribers located within the group and is responsible for directing calls to them [7]. Neighboring cells overlap each other to ensure the continuity of communications when the users move from one cell to another. Certain number of channels is allocated to each base station. A channel in the system can be thought of as a fixed frequency bandwidth (FDMA), a specific time-slot within a frame (TDMA), or a particular code (CDMA), depending on the multiple access technique used. BSs and MSCs take the responsibility of allocating channel resources to mobile stations [1].

The mechanism of transfer an on-going call from one cell to another as a user moves through the coverage area of a cellular system is called handoff. The handoff process starts when a handoff request is issued. The power received by the MS from BS of neighbouring cell exceeds the power received from the BS of the current cell by a certain amount called the handoff threshold. For successful handoff, a channel must be granted to handoff request before the power received by the MS reaches the receiver's threshold. Each handoff requires network resources to reroute the call to the new base station. If the number of handoff is less the switching load is less. If the channels are not available, handoff call is dropped resulting in poor QoS.

Each cell is equipped with a number of channels which are shared by new originating calls in the cell coverage area and the handoff calls. If no channel is available the call has to be blocked or queued or rejected depending on the call admission control schemes. The probability of the new originating call rejected is called Call Rejection probability and the probability that a handoff call rejected is called Handoff Rejection Probability. Generally the handoff request is initiated either by the Mobile Station or by the Base Station.

Channel assignment strategies can be classified into fixed, flexible and dynamic schemes [3]. Where there are no separate guard channels exclusively for handoff calls, it is fixed system. In fixed channel assignment (FCA) scheme, fixed numbers of channels are assigned to each cell and there are no guard channel reserved for handoff requests only. When a new call request or handoff request arrives, the base station will check to see if there is a channel available in current cell. The call will be allowed if there is a channel available and it will be dropped if no channel is available. So handoff request and new call request are dealt with equally priority. The cell doesn't differentiate between Handoff request and new call request. The channel assignment is done by First Come First Serve basis [9]. The Quality of Service is not satisfactory because the handoff blocking rate is same as new call blocking rate. The guard channels improve the probability of a successful handoff because they are reserved exclusively for handoff calls. The remaining channels can be shared equally between 
handoff requests and new calls [8][5]. Allocating guard channels for handoff calls improves the overall throughput. If the number guard channels are too big, the new call blocking rate will be high because several channels are set aside for handoff requests even when the traffic load is low. In this case, the resources are wasted by not serving either for handoff request or new call request. If the number is too small, the handoff blocking rate can't be guaranteed under high traffic load. So this scheme enhances the QoS by reducing the handoff blocking rate in a stable traffic load. While when the traffic load is changing periodically or dynamically due to big event or working rush hours, it is not flexible enough to get good QoS.

\section{PROPOSED SCHEME}

In this paper, an Adaptive Channel Allocation Scheme (ACAS) has been proposed in which the channels for handoff requests are dynamically allocated based on the observation of certain past period in the network. This scheme is aimed to utilize the available resources efficiently and also to balance the load in the network traffic.

The new call dropping rate determines the fraction of new calls that are rejected, while the handoff blocking rate gives the fraction of admitted calls that terminate prematurely due to handoff. Maximum resource utilization is the main objective of this research work. To get good resource utilization, less number of guard channels is assigned to the handoff calls when the number of handoff requests is less under the low traffic load. If more channels are saved for the handoff request in this condition, the resources are wasted because the channels don't serve either for handoff request or new call request. At the same time if the number of handoff requests is high then more number of guard channels are required to handle them. The balance of the new call rejection rate and handoff call rejection rate are monitored and maintained to get better resource utilization in cellular network.

\section{Adaptive Channel Allocation Scheme (ACAS)}

The selection of number of guard channels exclusively for handoff call is essentially important factor to get good Quality of Service. For different type of traffic load and mobility factor, different number of guard channels is needed to be allocated. The number of guard channels can't be fixed when the traffic load is changing with the time. This problem is addressed in the proposed scheme ACAS. The Adaptive Channel Allocation Scheme automatically searches the optimal number of guard channels to be reserved for handoff calls at each BS.

Consider the following parameters in a particular BS coverage area1.1.2, etc.

The total number of available channels $=\mathrm{C}$

Open Access Channels (new calls + Handoff calls) $=$ Co

Guard channels for handoff calls $=\mathrm{GCh}$

Where, $\mathrm{C}=\mathrm{Co}+\mathrm{GCh}, \mathrm{Co}=\mathrm{C}-\mathrm{Gch}$ and GCh is allocated dynamically

$\mathrm{Oc}=$ number of on-going calls on open access chanels

$\mathrm{Nc}=$ number of admitted new originating calls

$\mathrm{Hc}=$ number of admitted handoff calls

$\mathrm{H}=$ Total number of handoff call(admitted+rejected)

$\mathrm{Rn}=$ number of rejected new originating calls

$\mathrm{Rh}=$ number of rejected handoff calls

$\mathrm{t}=$ time period

$\mathrm{Th}=$ Threshold for handoff call rejection probability

Algorithm: ACAS (t,C ) // the algorithm takes time period and channels as input

\{

$\mathrm{Co}=\mathrm{C}-\mathrm{GCh}$

For every handoff call request Do

\{

if $\mathrm{Hc}<\mathrm{Gch}$ and $\mathrm{Hc}+\mathrm{Nc}<\mathrm{C} / *$ If channels are available in reserved category */

$\mathrm{Hc}=\mathrm{Hc}+1$ and grant admission

else If $\mathrm{Oc}<\mathrm{Co}$, then

$\mathrm{Oc}=\mathrm{Oc}+1$

$\mathrm{Hc}=\mathrm{Hc}+1$ and grant admission

Otherwise, $\mathrm{Rh}=\mathrm{Rh}+1$ and reject.

\}

For every new call request Do

\{

If $\mathrm{Oc}<\mathrm{Co}$, then 


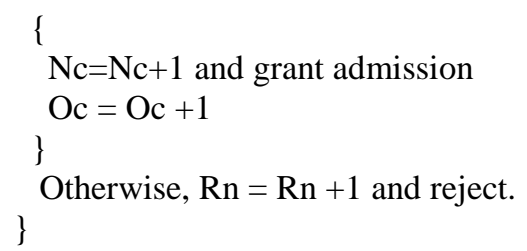

If a call is completed or handoff to another cell

\{

Check with MSC whether the ended call is handoff call or new originated call

If handoff call then

if $\mathrm{Hc}>\mathrm{Gch}$ then

$\mathrm{Oc}=\mathrm{Oc}-1$

$\mathrm{Hc}=\mathrm{Hc}-1$

Else

$\mathrm{Nc}=\mathrm{Nc}-1$

$\mathrm{Oc}=\mathrm{Oc}-1$

\}

If a handoff call is dropped and $\mathrm{Rh} / \mathrm{H}>=$ AuTh then

\{

$\mathrm{GCh}=\min \{\mathrm{GCh}+1, \mathrm{Cmax}\}$

IfRh/H $<=$ AdTh for $\mathrm{N}$ consecutive handoff calls, then

$\mathrm{GCh}=\max \{\mathrm{GCh}-1, \mathrm{Cmin}\}$

\}

$\mathrm{Nc}, \mathrm{Hc}$ are reported to monitor the successful handoff and new calls at a specifiedtimeperiod. \}//

The proposed algorithm increases the number of guard channels when a handoff call is dropped under the condition that $\mathrm{Rh} / \mathrm{H}>=\mathrm{Au} * \mathrm{Th}$, and it decreases the number of guard channels after a number of consecutive handoff calls under the condition that $\mathrm{Rh} / \mathrm{H}<=\mathrm{Ad} * \mathrm{Th}$. Au and Ad are usually chosen to be less than 1 . By choosing $\mathrm{Au}<1$, the algorithm will most likely keep the handoff blocking rate below its given threshold. The simulation studies are performed for comparisons of the proposed algorithm with fixed channel allocation (FCA) and static guard channel allocation policy. The result proves that the algorithm guarantees that the handoff dropping rate is below its given threshold and at the same time the new call dropping rate is minimized.

\section{Experimental Results}

The author simulates the algorithm in a six cells as a part of full network. The simulation program is implemented in Turbo C++, version 3.0, and run under MS DOS 6.2 environment. Object oriented approach is used to implement the real world environment. Results are directed to a text file and graph for the same is obtained using MATLAB. The comparison of results of the FCA scheme, static guard channel scheme, and the adaptive guard channel scheme has been made. The following are the initial parameters chosen for simulation.

$\mathrm{Oc}=0, \mathrm{Nc}=0, \mathrm{Hc}=0, \mathrm{Rn}=0, \mathrm{Rh}=0, \mathrm{H}=0$

$\mathrm{T}=10$ seconds $/ /$ time period for updating the measurements

$\mathrm{C}=20$ // No. of channels

$\mathrm{GCh}=10 \quad \mathrm{Au}=0.9 \quad \mathrm{An}=0.6 \quad \mathrm{Th}=0.2$

$\mathrm{N}=10 / /$ No. of consecutive calls

The following graphs show the comparative study of the three schemes, Fixed Channel Assignment without using guard channels, Static Guard Channel Assignment and Adaptive Guard Channel Assignment. 


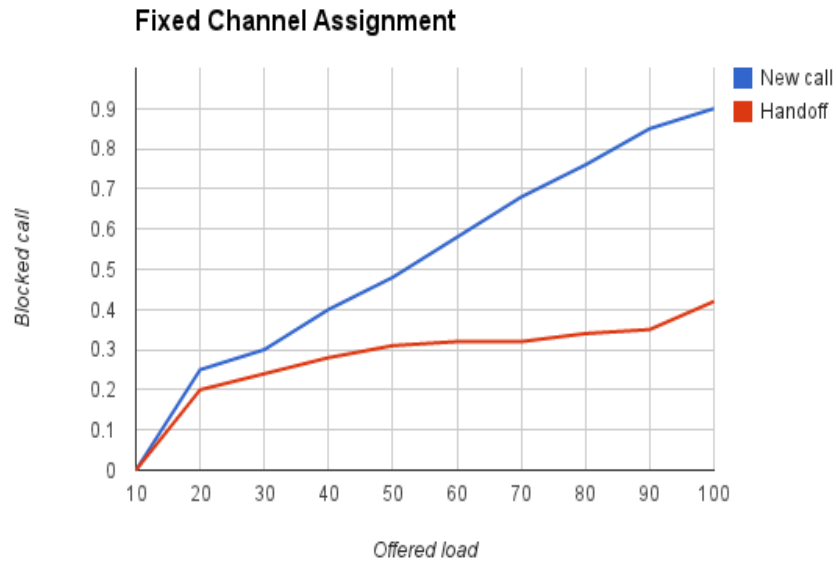

Offered load

Fig. 1.

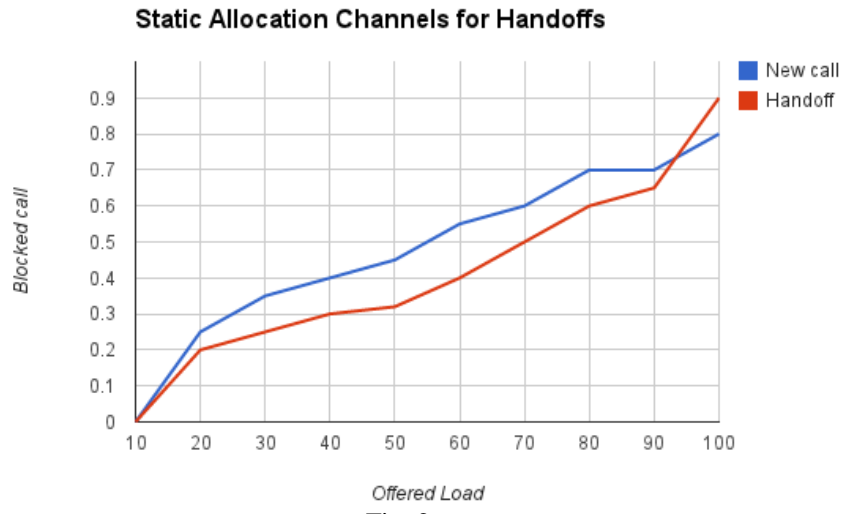

Fig. 2.

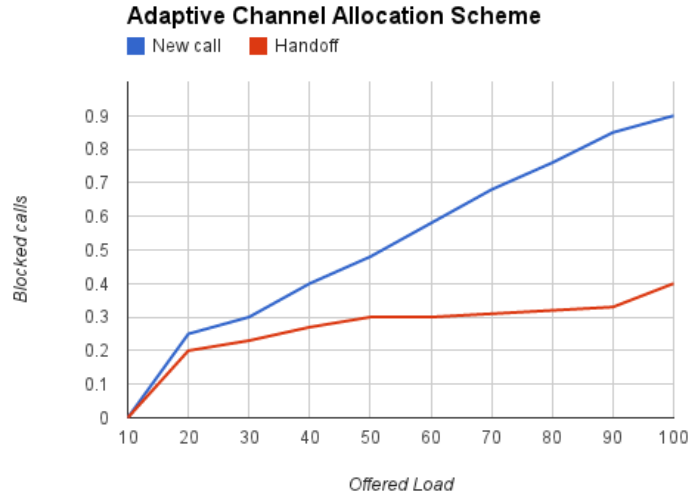

Fig. 3

Figure 1: Shows the simulated output of the FCA scheme where no guard channels are allocated for handoff calls. It simply works on the basis of FCFS (First Come First Served). The output graph shows that the handoff call rejection rate is slightly higher than the new originating call rejection rate. Handoff calls should be given higher priority than the new originating calls. The overall performance is also not satisfactory since both new call rejection and handoff call rejection ratio is comparatively high.

Figure 2: shows the simulated output of the static guard channel allocation scheme i.e., fixed number of guard channels exclusively allocated for handoff. Here the number of handoff call rejection is reduced but the new call rejection is highly increased because the number of guard channels allocated is high than which is actually required. Moreover in some cases if the number of guard channels is less, then handoff rejection rate will increase and hence affect the throughput.

Figure 3: shows the simulated output of our proposed scheme - Adaptive Channel Allocation Scheme (ACAS). Here the channels are not allocated static and they are allocated based on the traffic in the past certain period of time. The number of guard channels gets dynamically adjusted and it is clearly seen from the graph that both new calls and handoff calls utilizes the channel properly and the call rejection rate is low for both. Hence there is tradeoff 


\section{CONCLUSION}

A new Adaptive channel allocation scheme (ACAS) for cellular networks has been presented in this paper. Effective utilization of the available resources has been emphasized. The main problem faced in guard channel allocation is the number of guard channels chosen. The proposed algorithm adjusts the number of guard channels dynamically according to the dropping rate of handoff calls in a certain period of time. It keeps the handoff call rejection rate below the given threshold and it also reduces the new call rejection rate by decrementing the number of guard channels when it is observed to be more than needed. The QoS of proposed scheme is satisfactory.

\section{Journal Papers:}

\section{REFERENCES}

[1] S.Choi and K.G.Shin, Adaptive bandwidth reservation and admission control in QoS-sensitive cellular networks, IEEE Transection on Parallel and Distributed Systems, vol.13,no.9, pp.882-897,2002

[2] Nasif Ekiz, Tara Salih, Sibel Kucukoner and Kemal Fidanboylu, An overview of Handoff techniques in Cellular Networks, World Academy of Science, Engineering and Technology, 62005

[3] F. Siddiqui and S. Zeadally, Mobility Management across Hybrid Wireless Networks: Trends and Challenges, Computer Communications, vol. 29, no. 9, pp. 1363-1385, May 2006.

[4] Nasser, N., Hasswa, A. and Hassanein, Handoffs in Fourth Generation Heterogeneous Networks, IEEE Communications Magazine, vol. 44, pp. 96-103, 2006

[5] Rami Tawil, Jaques Demergian, Guy Pujolle, A Trusted Handoff Decision Scheme for the Next Generation Wireless Networks, IJCSNS International Journal of Computer Science and Network Security, VOL.8 No.6, June 2008

[6] V. S. Kolate, G. I. Patil, A. S. Bhide, "Call Admission Control Schemes and Handoff Prioritization in 3G Wireless Mobile Networks, International Journal of Engineering and Innovative Technology (IJEIT) vol.1, no.3, March 2012

\section{Books:}

[7] Qing-an-Zeng and Dharma P.Agarwal, Handbook of Wireless Networks and Mobile Computing (John wiley \& sons, Inc., 2002).

\section{Proceedings Papers:}

[8] Yi Zhang and Derong Liui, An Adaptive Algorithm for Call Admission Control in Wireless Networks, in Proceedings of the IEEE Global Communication Conference, San Antonio, TX,pp.3628-3632, November 2001

[9] I.Ramani and S.Savage. SyncScan, Practical fast handoff for 802.11 Infrastructure Networks, Proceedings of IEEE INFOCOM, March 2005

[10] Asd Malak Z. Habeib, Hussein A. Elsayed, Salwa H. Elramly and Magdy M. Ibrahim, Heterogenous Networks Handover Decision Triggering Algorithm Based on Measurements Messages Transfer using IP Option Header, The Seventh International Conference on Wireless and Mobile Communications - ICWMC 2011 